\title{
OPEN-LOOP HIRF EXPERIMENTS PERFORMED ON A FAULT TOLERANT FLIGHT CONTROL COMPUTER
}

\author{
Daniel M. Koppen \\ NASA Langley Research Center \\ Mail Stop 130 \\ 1 South Wright Street \\ Hampton, Virginia 23681 \\ USA
}

\begin{abstract}
During the third quarter of 1996, the Closed-Loop Systems Laboratory was established at the NASA Langley Research Center ( $\mathrm{LaRC}$ ) to study the effects of High Intensity Radiated Fields on complex avionic systems and control system components. This new facility provided a link and expanded upon the existing capabilities of the High Intensity Radiated Fields Laboratory at LaRC that were constructed and certified during 1995-96.
\end{abstract}

The scope of the Closed-Loop Systems Laboratory is to place highly integrated avionics instrumentation into a high intensity radiated field environment, interface the avionics to a real-time flight simulation that incorporates aircraft dynamics, engines, sensors, actuators and atmospheric turbulence, and collect, analyze, and model aircraft performance.

This paper describes the layout and functionality of the Closed-Loop Systems Laboratory, and the open-loop calibration experiments that led up to the commencement of closed-loop real-time flight experiments.

\section{LABORATORY FACILITIES \& LAYOUT}

During the third quarter of 1996, the Closed-Loop Systems Laboratory was established to study the effects of High Intensity Radiated Fields (HIRF) on complex highly integrated avionic systems. A modular design was chosen to facilitate a flexible experimental layout. Three functional modules were defined as shown in Figure 1. Each part can be shifted, expanded, or deleted without affecting the other parts. However, each part is linked with the other and it is these interfaces which truly set the limits of functionality within the Closed-Loop Systems Laboratory (CLSL).

\section{Radiation Generator}

The first task was to model the electromagnetic testing environment. Since most of the testing was to be done in the HIRF Laboratory, this meant blocking out the general functional modules that made up the Radiation Generator. A functional diagram of this module is shown in Figure 1.

In the specific case of the HIRF Laboratory [1], the Radiation Environment is the reverberation chamber. Within the chamber are both transmitting and receiving antennas as well as any sensors that might be needed for a specific test. The components of the Signal Generation and Measurement module consist of a synthesized sweeper for signal generation, a network analyzer, a spectrum analyzer, an oscilloscope, and a high power amplifier. These devices are software controlled within the Environment Controller module by using Hewlett Packard's visual engineering environment (VEE) control software and an IEEE 488 bus interface.

These same general functions apply to Bulk Cable Injection testing. Again, referencing Figure 1, the Radiation Environment consists of a copper clad 


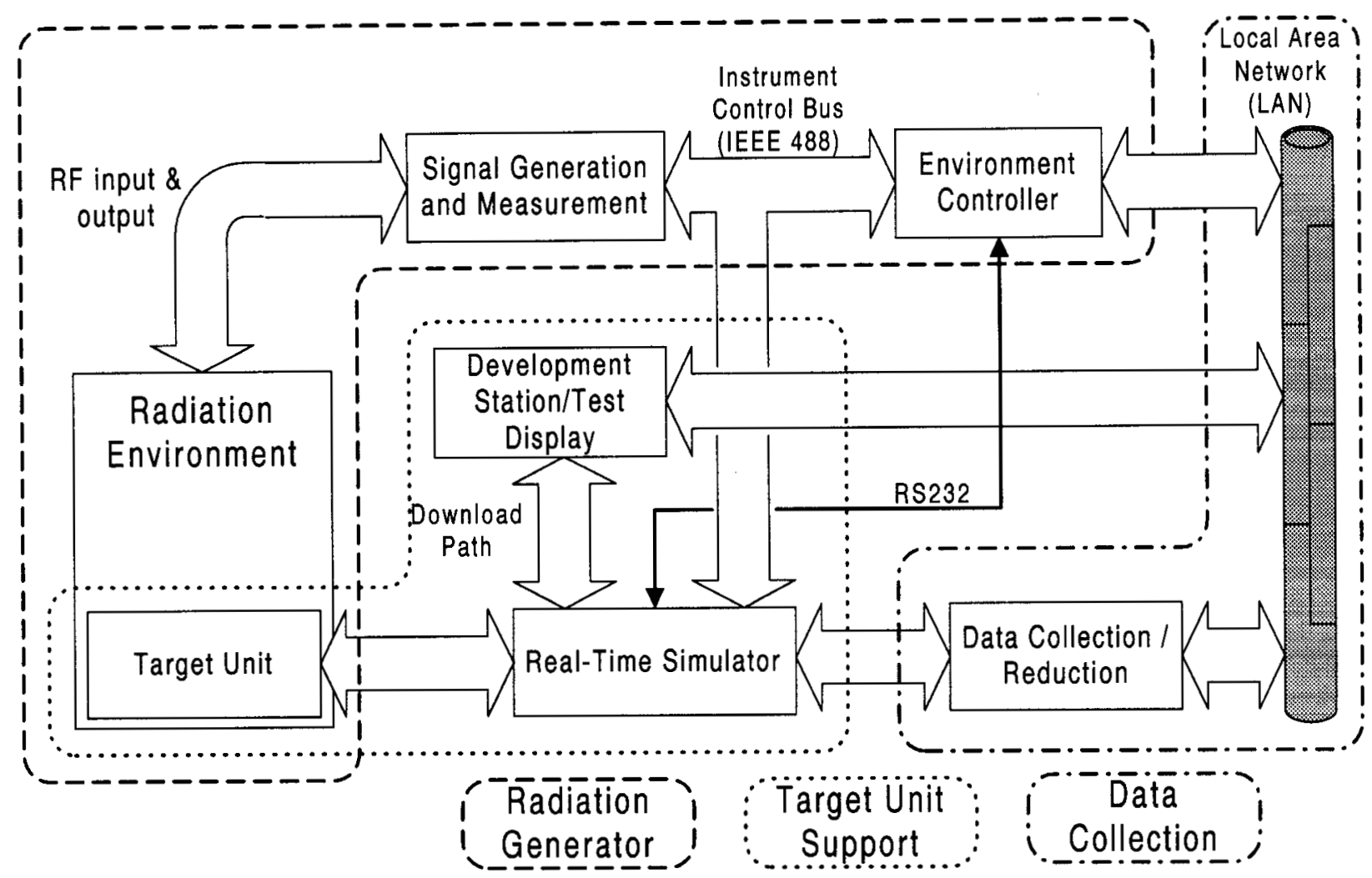

Figure 1 : Function Diagram of Closed-Loop Systems Lab Modules

grounded table with an injection probe, current probe, and Line Impedance Stabilization Network (LISN). The environment is controlled and measured from the Signal Generation and Measurement block which consists of two polynomial waveform synthesizers, a spectrum analyzer, an oscilloscope, and a low power amplifier. Again, the IEEE 488 bus was used for instrument control. However, National Instrument's LabView rather than HP's VEE was chosen as Environmental Control Software due to a broad user base at NASA's Langley Research Center.

Functional Differences in Radiation Generator Modules. The reverberation chambers within the HIRF Laboratory provide near homogeneous randomized electromagnetic fields and place the entire Target Unit in the test environment. The Bulk Cable Injection section of the CLS Lab provides localized fields for debugging Target Unit and Real-Time Simulator code and localized component tests. After running a formal test in the HIRF reverberation chamber, and analyzing the data for frequencies of sensitivity, injecting low power signals at these frequencies within the flight computer targets individual components that could potentially cause deviant behavior. In addition, through the use of the dual polynomial waveform synthesizers, waveforms that simulate lightning induced transients can be emulated.

The primary interface to the Target Unit Support module, is the IEEE 488 bus. This allows the Environment Controller to treat the entire module as just another instrument and incorporates very tight control of an automated experiment. However, this requires a complete surrender of experiment control to the Environment Controller and a complete understanding by the Controller 
program of legitimate command sequences. For more localized experiment control with less synchronization, an RS232 interface has been implemented to send an asynchronous signal to the Environment Controller to flag events-ofinterest during the test and maintain controller/simulator isolation. Experiments can also be performed with complete independence by using wireless headsets to coordinate experiment events with radiation conditions.

\section{Target Unit Support}

The second task was to define the Target Unit Support functions. Again, Figure 1 shows a functional module of a closed-loop experiment.

The Target Unit would be the flight computer. This computer is placed in the radiation environment and subjected to various frequencies and power levels of electromagnetic radiation. The Real-Time Simulator is a support computer located outside the radiation environment that runs a real-time simulation so that the Target Unit is exercised over its operating envelope. In addition, the Real-Time Simulator is the first line in data collection, since during testing, it has a direct line to the Target Unit. These two units form the basis for any closed-loop test. The Development Station/Test Display is where the coding for the simulation and Target Unit are done prior to testing. This station provides a coding environment, a cross compiler for the target computing system, and a download engine. During the actual test, the Development Station is inert, so the unit can be assigned a secondary role as a Real-Time Test Display for parameters of interest. Usually this display shows red line limits on Target Unit signals so a rapid shutdown could be initiated if necessary.

The LAN/Internet Interface is an ethernet connection that allows experiment designers to edit/compile code on local systems, then download it to the Development Station for crosscompiling or, if it is already in executable form, to download directly to the Real-Time Simulator. This functional interconnect makes it possible to custom design an experiment remotely and write the code for the hardware available in the CLSL. Finally, there is an interface between the RealTime Simulator and the Data Collection module which links to the Data Collection/Reduction/Archiving tools. In the experiment described later in this paper, this is a portion of dual-ported memory that is shared between the Real-Time Simulator and the Data Collection computer. This provides the capability for on-line analysis of data during an experiment. To date, however, analysis is performed during post-test processing and the dual-port memory is used to store experimental data that is converted into computer files after each test run. Current experiments incorporate enough memory in the Real-Time Simulator to store an entire flight. The entire data set is then downloaded via the LAN/Internet to the machine of choice.

\section{Data Collection}

The third module consists of Data Collection/Reduction/Archiving. This is a combination of hardware and software tools to analyze and reduce the collected data. Currently the hardware support includes 3 Gbytes of hard disk space, a Sun 4/360 with a VME backplane and LAN/Internet connection, a 1 Gbyte removable hard drive system, and a CD-ROM writer for archival storage. Software tools include MATLAB and PV-Wave for modeling and numeric analysis, and a database for tracking experiments. The LAN/Internet connection allows researchers to receive their data within minutes of a test run. However, to avoid network congestion, local data reduction is recommended. 


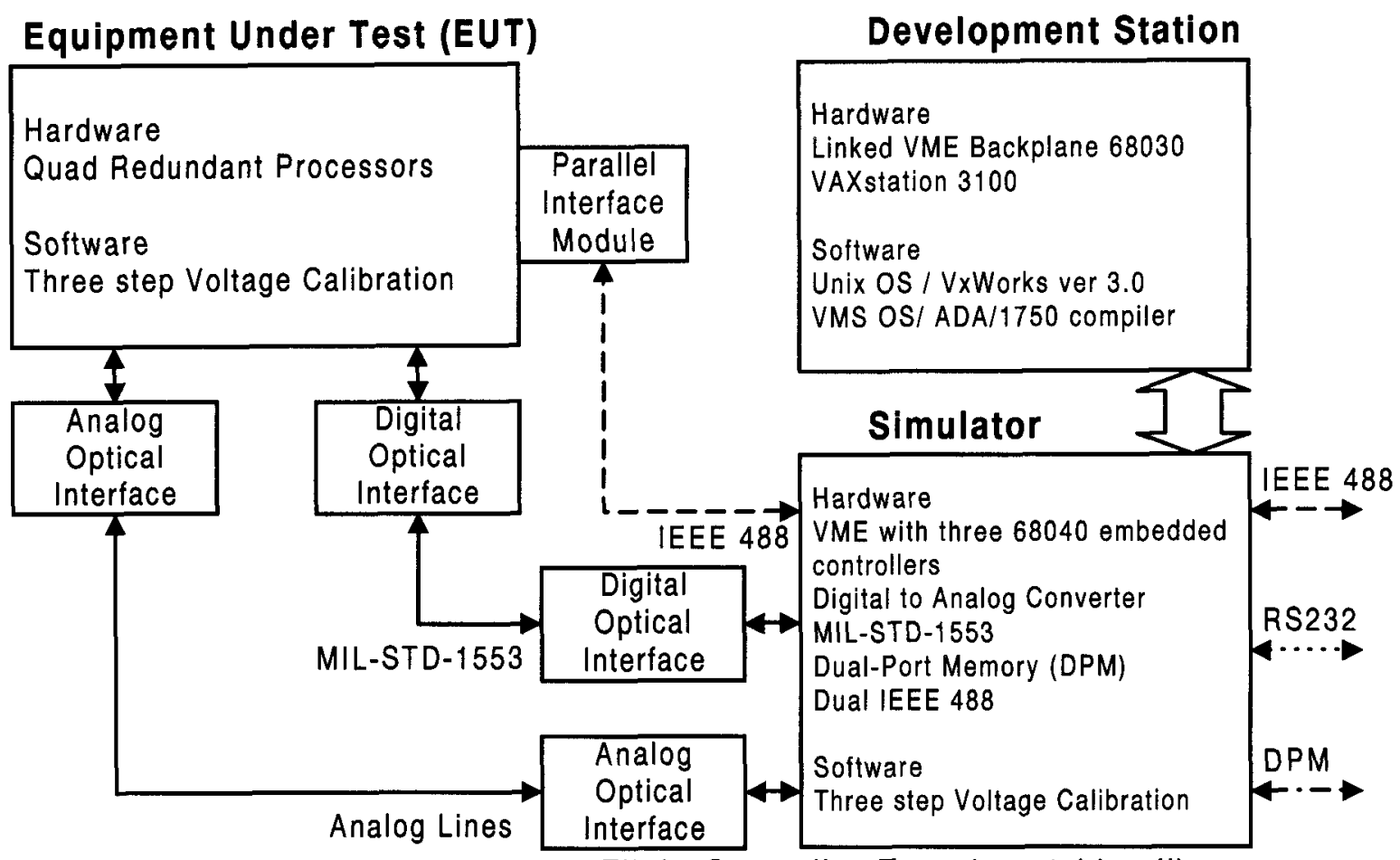

Figure 2 : Fault Tolerant Flight Controller Experiment (detail)

\section{HARDWARE FOR QUAD-REDUNDANT FAULT TOLERANT FLIGHT CONTROLLER EXPERIMENTS}

For the first experiments, the Target Unit was a fault tolerant flight control computer with quadredundant 1750 microprocessor-based computer modules. This unit, although digital in design, receives sensor inputs and sends actuator command outputs via analog voltages. In addition, a MIL-STD-1553 interface can be used for digital input/output to close the control loop. The specific Experiment Layout for this unit can be seen in Figure 2.

The Equipment Under Test (EUT) consists of four independent modules. Each module has a 1750 processor, 48 Kbytes of EPROM, 2 Kbytes of scratchpad RAM, and 8 Kbytes of sharable RAM. All $\mathrm{I} / \mathrm{O}$ is memory mapped into a 2 Kbyte space. For programming the EPROM, a special Parallel Interface Module (PIM) is attached and is controlled using an IEEE 488 interface from the Simulator. During testing, the PIM is removed.

The Analog Optical Interface is a set of custom built circuits that convert all of the electrical analog/discrete inputs and outputs from the EUT into light that can be transmitted down a fiber optic link. This permits a safe, noise-free method of transmitting the signals in and out of the reverberation chamber. However, since each line converter is tuned to the individual loading characteristics of the analog line coming from the flight controller, the Analog Optical Interface becomes "married" to the EUT. No independent testing of this box can be done. For this reason, every effort to insure radiation tightness was implemented on the EUT side of the link.

The Digital Optical Interface is a MIL-STD-1553 fiber optic converter purchased from Cybernetic Evaluation, Inc. The EUT side is radiation tight. 


\section{Simulator}

The Simulator is based on a 20 slot VME backplane. The system consists of 3 Motorola 68040 based real time controllers, 5 digital to analog converter (DAC) boards, 1 analog to digital converter (ADC) board, 1 MIL-STD-1553 interface board, and 1 dual-port memory interface card. The 5 DAC's and 1 ADC link the simulator to the Analog Optical Interface, the 1553 board links to the Digital Optical Interface, and the dualport memory is linked to the Data Collection Module. Even with 5 DAC's and an ADC only one set of I/O lines can be supported, so the Analog Optical Interface performs a fan out function so that all four of the EUT's processors receive the same inputs. In addition, only one of the 3 real-time processors has access to the DAC's, so the entire simulation is run through a single controller. The other two controllers are used for monitoring and debugging.

\section{Development Station}

The Development Station for this experiment is unique. The Simulator Development Station is actually embedded into the same 20 slot backplane as the Simulator and uses VxWorks to provide a C/68040 compiler environment. However, it is an independent UNIX computer system that talks to the runtime processors using TCP/IP over the backplane. The EUT Development Station is a VAX/VMS system with an $\mathrm{ADA} / 1750$ compiler. After being complied, the download path includes sending the code over

\begin{tabular}{|l|c|c|}
\hline & +15 to $-15 \mathrm{v}$ & +10 to 0 volts \\
\hline Maximum & +15 & +10 \\
\hline $50 \%$ of $\max$ & +7.5 & +5 \\
\hline zero & 0 & 0 \\
\hline $50 \%$ of $\min$ & -7.5 & 0 \\
\hline minimum & -15 & 0 \\
\hline
\end{tabular}

Table 1 : Critical point values for typical analog signal lines the ethernet to the Simulator Development Station, which in turn, downloads it through the IEEE 488 interface to the EPROM Parallel Interface Module.

\section{SOFTWARE FOR QUAD-REDUNDANT FAULT TOLERANT FLIGHT CONTROLLER EXPERIMENT}

After assembling the hardware described above, a process was needed to determine experimental limits for this setup. Two questions needed an answer. 1) Are both Optical Interfaces sufficiently radiation hardened to discount their effect on experimental data? 2) Can a radiation zone of probable EUT upset be defined without destroying the unit?

To answer these two questions, a special calibration procedure was developed. The maximum and minimum voltage was calculated for each analog signal line. Five critical points were then defined. They are 1) maximum voltage, 2) $50 \%$ of maximum voltage, 3) the zero point, 4) $50 \%$ of minimum voltage, and 5) minimum voltage. Table 1 illustrates an example.

The Simulator software was developed to hold all analog voltages at the $50 \%$ of maximum level. The EUT software on each of the four processor modules takes 1,000 samples of the signal lines and sends the values back to the simulator through the Digital Optical Interface. These values, along with the original reference voltage, are stored in dual-port memory. The Simulator then changes the analog voltage to the 0 level and the process repeats for another 1,000 samples. Finally, the $50 \%$ of minimum voltage is applied and the last 1,000 samples are collected. The maximum and minimum values are never used so that if the induced radiation adds to the applied voltage, specified internal voltages will not be exceeded. The resulting 3,000 line data file is then stored on the Data Collection machine and the dual-port memory is cleared. The first four lines of a 
sample data file are shown in Table 2.

The first column of Table 2 represents the reference voltage for the signal line and the remaining four columns are the values for each of

\begin{tabular}{|c|c|c|c|c|}
\hline REF. & $\mu \mathrm{P} 1$ & $\mu \mathrm{P} 2$ & $\mu \mathrm{P} 3$ & $\mu \mathrm{P} 4$ \\
\hline 5.000 & 4.966 & 4.966 & 4.951 & 4.976 \\
\hline 5.000 & 4.961 & 4.971 & 4.971 & 4.956 \\
\hline 5.000 & 4.961 & 4.956 & 4.971 & 4.961 \\
\hline 5.000 & 4.976 & 4.956 & 4.961 & 4.956 \\
\hline
\end{tabular}

Table 2 : First four lines of a data file for a single signal line

the four processor modules. With no external radiation present, the data gives us a statistical model of the system's standard voltage drifts.

\section{Optical Interface Tests}

Once the nominal characterization data was collected, a test plan was developed to determine if the Optical Interfaces had sufficient shielding from electromagnetic radiation. Testing began by placing the EUT in a reverberation chamber with a continuous wave frequency of $250 \mathrm{MHz}$ and a peak field strength of 50 volts/meter. The above procedure of collecting 3,000 samples for each signal line was followed. The peak field strength was increased in 50 volts/meter steps until a 200 volts/meter peak field strength was achieved. The $250 \mathrm{MHz}$ signal was then reset to $50 \mathrm{~V} / \mathrm{m}$ and amplitude modulated with a progression of square wave frequencies at $39 \mathrm{~Hz}, 1 \mathrm{KHz}, 25 \mathrm{KHz}, 100$ $\mathrm{KHz}, 350 \mathrm{KHz}$ and $750 \mathrm{KHz}$. Again, each modulated frequency was increased in $50 \mathrm{~V} / \mathrm{m}$ steps. The carrier frequency was increased in 100 $\mathrm{MHz}$ steps, and the entire sequence was repeated to a frequency of $950 \mathrm{MHz}$. However, as shown in Table 3, different peak field strengths were attained for each carrier frequency.

During the experiment, one of the spare 68040 processor boards in the simulator monitored the data in real-time for voltages that were beyond a predetermined limit. A limit of $1 \%$ beyond normal drift was chosen for the first test.

$\begin{array}{cc}\begin{array}{c}\text { Carrier Frequency } \\ (\mathrm{MHz})\end{array} & \begin{array}{c}\text { Field Strength } \\ (\mathrm{V} / \mathrm{m})\end{array} \\ 250 & 200 \\ 350 & 200 \\ 450 & 400 \\ 550 & 600 \\ 650 & 600 \\ 750 & 600 \\ 850 & 600 \\ 950 & 600\end{array}$

\section{Table 3 : Peak Field Strengths for each Carrier} Frequency

During the experiment, the $1 \%$ limit was exceeded only at $250 \mathrm{MHz}$ and $550 \mathrm{MHz}$ nonmodulated. At $250 \mathrm{MHz} 200$ peak $\mathrm{V} / \mathrm{m}$, a single analog line connected to the first processor module with a full scale voltage of +750 millivolts to -750 millivolts violated the $1 \%$ limit 18 times at $+50 \%, 88$ times at $0 \%$, and 90 times at $-50 \%$ with a maximum 9 millivolts of induced voltage. At $550 \mathrm{MHz} 600$ peak $\mathrm{V} / \mathrm{m}$, three lines all connected to the third processor module failed the $1 \%$ limit 9 times at $+50 \%, 7$ times at $0 \%$, and 3 times at $-50 \%$. These were all +10 volt to -10 volt lines and incurred a maximum induced voltage of 69 millivolts. With only these minor limit violations, both of the Optical Interface units were deemed to be acceptably EM hardened.

\section{Static Flight Controller Tests}

To define a zone of probable upset without destruction, a simple modification of the Flight Controller was needed. At the connector of each of the cable interfaces, is a passive filter that reduces the amount of electromagnetic energy coupled into the unit. There is one at each connector of the cable assembly. By selectively removing the filters on the Flight Controller 
processor modules, electromagnetic energy can be coupled directly into the module using the electrical interface lines as antennas. Using the same procedure as before, data was collected by removing filters from each of the individual modules of the EUT. Then all filters were removed from the EUT side of the cable, with the exception of the power supply, and an entire data set was collected for the fifth time. After the fifth data set was collected, a final data set was collect with no electromagnetic radiation present and compared to the initial no-EM data set to verify that no permanent damage had occurred. In addition, a closed-loop autoland simulation was run to verify proper operation of the EUT/Simulator module.

The data collected from these tests is currently being analyzed to find a function which describes the relation between the chamber peak field strength and voltage coupling into the Flight Controller for each frequency. Comparing this function to the upper limit of voltage/current specifications for internal flight controller chips, should give an upper destructive limit on the EUT.

\section{Future Plans \& Tests}

Even though upper destructive limits have not been established, dynamic flight tests have already begun within the frequencies and field strengths defined above [2]. In addition, experiments involving a modified commercial flight controller are being planned [3]. Future plans include replacing some of the simulated sensors and actuators with avionics hardware and placing them in the reverberation chambers to enhance our understanding of electromagnetic effects on the entire system. Ultimately, a computer model will be built to explore methods for detecting and avoiding electromagnetic upsets, which would result in safer flight for future aircraft.

\section{REFERENCES}

[1] Rueben A. Williams, "The NASA HighIntensity Radiated Fields Laboratory," AIAA/IEEE $16^{\text {th }}$ DASC Proceedings, October 1997

[2] Dr. Celeste M. Belcastro, "Closed-Loop HIRF Experiments Performed on a Fault Tolerant Flight Control Computer," AIAA/IEEE $16^{\text {th }}$ DASC Proceedings, October 1997

[3] Richard Hess, "Robust Operation in the Presence of Lightning and Other Electromagnetic Threats", AIAA/IEEE $16^{\text {th }}$ DASC Proceedings, October 1997

Disclaimer: The use of brand names is for completeness and does not imply NASA endorsement. 